\title{
Informed successive condition assessments in bridge maintenance
}

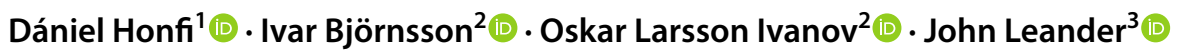

Received: 15 December 2019 / Revised: 17 May 2020 / Accepted: 3 June 2020 / Published online: 18 June 2020

(c) The Author(s) 2020

\begin{abstract}
The condition assessment of bridges considers a combination of information from different sources rendering multiple levels of assessment possible. This paper illustrates how successive condition assessment strategies increase the expected utility compared to single choice decisions through Bayesian inference. Multiple levels of assessment allow for additional possibilities for obtaining structural health information and updating one's beliefs about structural condition. Thus, more informed decision-making is possible with respect to the gain in accuracy versus the costs of the assessment options. The paper aims to introduce how the successive approach can be implemented and in which scenarios it provides an increase in expected utility in comparison to one instant decision. To highlight this, a few pedagogical numerical examples are provided.
\end{abstract}

Keywords Bridge maintenance $\cdot$ Condition assessment $\cdot$ Informed decision making $\cdot$ SHI $\cdot$ VoI

\section{Introduction}

Our infrastructure is aging. In Sweden, for instance, the average age of all nationally owned bridges is about 50 years [24]. Studies in both Europe and the United States have highlighted the continual need for rehabilitating existing bridges [5, 25]. The more recent and sudden collapse of the Morandi bridge in Genoa, Italy, has in a spectacular way, raised this issue even further $[4,5]$.

The successful management of infrastructure assets such as bridges requires reliable structural health information (SHI) concerning the actual condition of these structures. In fact, the condition of infrastructure assets is assessed on a regular basis to support maintenance decisions. These assessments may be carried out in a number of different ways and may be based on existing (sometimes old) information, visual inspections, on-site measurements, monitoring systems, etc. [22]. If the assessment yields doubts concerning the condition of the asset, then resources can be spent

Dániel Honfi

daniel.honfi@ri.se

1 Building Technology, RISE Research Institutes of Sweden, 40022 Gothenburg, Sweden

2 Division of Structural Engineering, Lund University, 22100 Lund, Sweden

3 Department of Civil and Architectural Engineering, KTH Royal Institute of Technology, 10044 Stockholm, Sweden to either repair, strengthen or replace the damaged/faulty components.

The appropriateness of such typically costly actions is, however, dependent on the validity of the SHI which underlies any condition assessments as well as the verification systems used [7]. As an alternative to costly maintenance actions, enhanced conditions assessments may be carried out to obtain a more accurate representation of the severity of damage in a structure. Such assessments reduce any inherent conservativeness and uncertainty associated with more simple condition assessments: (1) using a more advanced verification format; (2) employing a more sophisticated structural model, i.e. reducing modelling uncertainties; and (3) reducing epistemic uncertainties in model inputs e.g. by measurements of loads, material properties or other relevant structural parameters [7]. The aim of these strategies is to improve the sensitivity/specificity of the assessments [11]; i.e. the method's ability to adequately capture the true state of the structure (considering all uncertainties involved).

In some previous work, Goulet et al. [6] provide a preposterior analysis framework to utilize SHI. The framework focuses on determining the optimal sequences of future measurement and intervention actions (by minimizing the expected costs while fulfilling reliability constraints) and quantifying the potential benefit of making measurements. Their framework is directly applicable in industries where reliability analysis, and the collection of empirical data underlying these analyses, is common practice. In the 
condition assessment of common bridges, however, probabilistic assessment is rarely used. Therefore, a rational and systematic decision support framework is proposed in [2], with the aim of being applicable for practical cases, where deterministic approaches are used to verify structural performance. The framework enables to select the best assessment option and subsequent intervention if several alternatives with different costs and efficiency are available. The framework has been applied on other practical cases of bridges in Sweden $[13,19]$.

The current paper builds on [2] by investigating the implications of sequential decision making for maintenance decisions. In practice, the condition of a structure may be determined in a sequential process where the level of assessment and expected accuracy is increased at each progressive step. The accuracy of subsequent levels of assessment is then a result of obtaining and incorporating more useful SHI in the safety verification $[3,18]$. Initial doubts about the state of the structure may be based on simple desktop assessments using codified verification format. However, the allocation of limited resources for costly repair or strengthening actions may be spared if additional, more enhanced, assessments are made. This process may be repeated in certain instances where additional information is used to update model predictions leading to a sequential and reflective process. While the approach in [2] accounts for the suitability of adopting enhanced assessments in lieu of costly invasive actions, the current paper extend these considerations towards informed successive condition assessments and subsequent decision making. The term successive refers to the possibility of updating knowledge about the real condition more than once, e.g. information (about the same state) could be collected or structural analysis could be performed in successive steps, typically at different levels of assessment. By informed, the authors mean that the updating of knowledge is based on evidence, formally by using Bayesian inference. It should be mentioned that the simple approach presented here does not explicitly consider changes in condition over time due to deterioration. Thus, it is only seen as a single point-in-time updating as opposed to sequential updating approaches [20] considering time aspects. Nevertheless, a further temporal extension using dynamic Bayesian networks is possible; however, it is out of the scope of this paper.

\section{$2 \mathrm{SHI}$ in bridge maintenance}

\subsection{Successive condition assessments and SHI}

In practical bridge management, the decision maker typically aims to find an optimal allocation of the available resources for a population of bridges. A simple strategy is trying to postpone expensive interventions if it seems reasonable and could be justified. This is typically possible if the initial structural assessment indicates a minor safety deficit and the cost of more detailed assessments is low in relation to the cost of possible maintenance actions. In a number of cases, detailed assessments are carried out in an iterative manner as suggested by, e.g., Kühn et al. [12] and in ISO 13822 [10]. First a less expensive, less sophisticated method is used. If no improvement, in terms of increasing the safety margin, is observed, no more time and resources are wasted for detailed assessments and a decision is made on whether to repair, strengthen or replace. Similarly, if the first iteration shows acceptable structural performance, i.e. the improvement is significant, then no further actions are required. In the intermediate case, where some improvement in the safety level is observed, yet not sufficient, further, more detailed assessments could be utilized. The results of prior assessments, in essence, provide additional and potentially valuable SHI to subsequent more enhanced levels of assessments. The implication of this for the potential cost savings in the maintenance of bridges requires closer attentions.

\subsection{Vol in bridge maintenance}

An important aspect of condition-based maintenance is that decisions about interventions are made using predictions about future performance. These predictions are based on SHI obtained from the existing structure. The process entails two procedures: (1) updating (the imperfect) knowledge about structural health for better predictions (model updating); and (2) making decisions on allocation of resources for maintenance interventions to maximize expected utility (decision making). Both procedures involve SHI as depicted in Fig. 1 and are interlinked as indicated by the dashed arrows.

Ideally, the updating and decision-making procedures are handled in a common and unified framework [15] to maximize the value of SHI; i.e. even decisions concerning inspections, monitoring systems and campaigns, and the predictive model itself aim to maximize expected

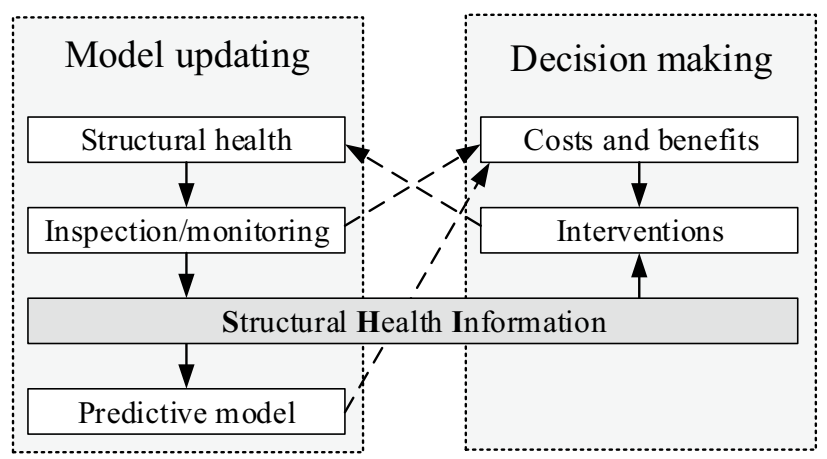

Fig. 1 SHI as part of model updating and decision making 
utility. However, in common bridge management systems (BMS) these aspects are traditionally treated separately for practical reasons. An obvious strength of a common framework is that the value of information (VoI) can be assessed explicitly, and thus included in the expected value maximization process. VoI-analysis focuses on evaluating the expected gain from reducing uncertainty through experiments (or more generally through directly studying the actual behavior of structures). The analysis is based on Bayesian statistics where probability represents degrees of belief rather than relative frequencies. The prior beliefs about plausible values (of parameters, event likelihoods etc.) are updated based on likely values drawn from sampled data (likelihood functions) to form a posterior belief using Bayes theorem (posterior analysis).

In recent years, VoI-analysis has gained significant popularity in relation to inspection planning and monitoring of structures. For example, Straub [21] presented an efficient framework for the determination of the VoI when information is collected to improve the structural system reliability and illustrated its application for fatigue monitoring. Similarly, Thöns [23] introduced an approach for the quantification of the value of structural health monitoring (SHM) to provide a rational basis for the optimization of the structural risk and integrity management considering utility gains via SHM and inspection information.

In VoI-analysis the prediction of the likelihood function is made conditional on the prior beliefs to obtain the expected posterior distribution, i.e. the outcome of the experiments is predicted based on current knowledge. In the so called preposterior analysis, these are then combined with the current knowledge to predict the state of knowledge after the experiments had been carried out [26]. The preposterior analysis is useful for understanding whether it is worth, in an expected utility context, to carry out any given experiment.

If the question is to determine the experiment $e$ with the highest expected utility $u^{*}$ (for the fundamental case presented in 3.1), it could be selected according to:

$u^{*}=\max _{e}\left(\sum_{z \in Z} P_{z \mid e}\left(\max _{a}\left(\sum_{\theta \in \Theta} u(e, \tilde{z}, a, \tilde{\theta}) P_{\theta \mid z}^{\prime \prime}\right)\right)\right)$

where $P_{z l e}$ is the marginal probability of outcome $z$ (for given experiment $e$ but for unspecified structural condition $\theta$ ), and $P_{\theta \mid z}^{\prime \prime}$ is the posterior conditional probability of state $\theta$ given the experimental outcome $z$. Furthermore, $u(e, z, a, \theta)$ refers to the utility of performing a particular experiment $e$, observing a particular outcome $z$, taking a particular action $a$ and then finding a particular state of the bridge $\theta$. The tilde in the previous notations is used to distinguish random variables from particular values. For a more detailed description about the topic, the reader should refer to $[1,17]$.
VoI is the expected gain that would result from the reduction of uncertainty due to additional information obtained through an experiment $[8,9]$. In other words, it represents the maximal amount at which the decision maker would be willing to buy an experiment (to obtain more information) [14]. Mathematically, it can be defined as the difference between the maximum expected utility with and without obtaining additional information, i.e. conducting an experiment:

$\mathrm{VoI}=u^{*}\left(e_{i}\right)-u^{*}\left(e_{0}\right)$

where $u^{*}\left(e_{i}\right)$ is the maximized (by appropriately selected action $a$ based on outcome $z$ ) expected utility when selecting experiment $e_{i}$, and $u^{*}\left(e_{0}\right)$ is the maximized expected utility when choosing no experiment $e_{0}$.

\section{Informed decision-making framework}

\subsection{Fundamental case}

In [2] an optimal assessment method, as well as subsequent interventions, are evaluated using a Bayesian decision making framework. The assessment methods, which incorporated the possibility for collecting more structural health information, are then considered as possible experiments for obtaining new useful information about the condition of the structure. A fundamental and simplified decision model was used to exemplify this and, for the purposes of completeness, will also be presented in this paper (although using different input values).

The decision problem is presented by the influence diagram in Fig. 2, where $E$ refers to the set of possible experiments (assessment options), $C_{E}$ are the costs of the experiments, $Z$ denotes the experiments' possible outcomes (the results of the assessments), $A$ represents the intervention actions, $\Theta$ stands for the possible states of nature, (i.e. the

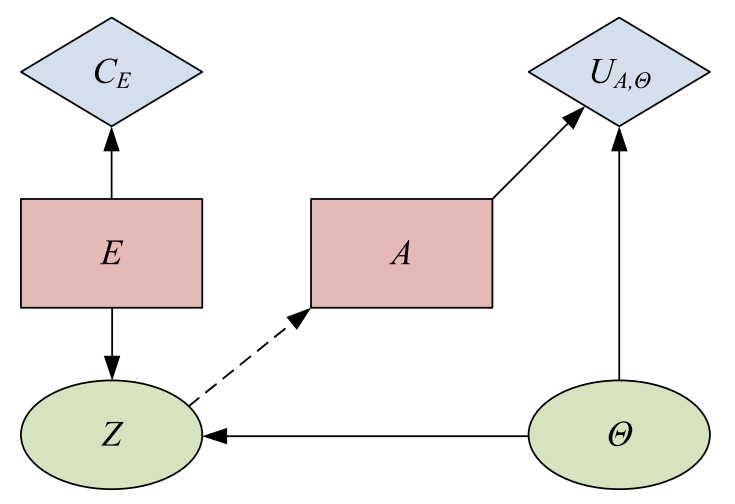

Fig. 2 Influence diagram for the fundamental case 
Table 1 Sample likelihoods

\begin{tabular}{|c|c|c|c|c|c|c|c|c|}
\hline \multirow[t]{3}{*}{$Z$} & \multicolumn{8}{|l|}{$E$} \\
\hline & \multicolumn{2}{|l|}{$e_{0}$} & \multicolumn{2}{|l|}{$e_{1}$} & \multicolumn{2}{|l|}{$e_{2}$} & \multicolumn{2}{|l|}{$e_{3}$} \\
\hline & $\theta_{1}$ & $\theta_{2}$ & $\theta_{1}$ & $\theta_{2}$ & $\theta_{1}$ & $\theta_{2}$ & $\theta_{1}$ & $\theta_{2}$ \\
\hline$z_{0}$ & 1 & 1 & 0.0 & 0.0 & 0.00 & 0.00 & 0.000 & 0.000 \\
\hline$z_{2}$ & 0 & 0 & 0.9 & 0.1 & 0.99 & 0.01 & 0.999 & 0.001 \\
\hline$z_{3}$ & 0 & 0 & 0.1 & 0.9 & 0.01 & 0.99 & 0.001 & 0.999 \\
\hline
\end{tabular}

Table 2 Prior failure probabilities

\begin{tabular}{lll}
\hline Damage severity & $P_{\theta_{1}}^{\prime}$ & $P_{\theta_{2}}^{\prime}$ \\
\hline Minor & 0.99999 & 0.00001 \\
Moderate & 0.9999 & 0.0001 \\
Significant & 0.999 & 0.001 \\
Severe & 0.99 & 0.01 \\
\hline
\end{tabular}

bridge's actual condition), and $U_{A, \Theta}$ is the utility associated to given $a \in A, \theta \in \Theta$ combinations.

The possible choices for the decision and chance nodes are as follows: $E=\left\{e_{0}, e_{1}, e_{2}\right\}=\{$ No Assessment; Assessment 1; Assessment 2 $\}, \quad A=\left\{a_{0}, a_{1}\right\}=\{$ Do Nothing; Repair $\}$, $Z=\left\{z_{0}, z_{1}, z_{2}\right\}=\left\{\right.$ Dummy; Outcome more favourable to $\theta_{1}$; Outcome more favourable to $\left.\theta_{2}\right\}, \Theta=\left\{\theta_{1}, \theta_{2}\right\}=\{$ Sufficient; Insufficient $\}$.

The costs of various types of experiments are $C_{E}=\{0 ;-10 ;-200\}$ and the utilities $U_{A, \Theta}=\left\{0 ; C_{f}\right\}$ for $a_{0}$ :'Do Nothing' and $\left\{C_{r} ; C_{r}\right\}$ for $a_{1}$ :'Repair'. $C_{r}=-1000$ is the cost of repair, while the cost of failure $C_{f}$ in this paper is considered as a studied parameter $\{10100100010000\} C_{r}$ accounting for the critically of the bridge, respectively \{Low; Mid-low; Mid-high; High\}.

The sensitivity/specificity of the assessment methods is characterised by their sample likelihoods, i.e. the conditional measures on $Z$, given in Table 1 . Note that $e_{3}$ is not considered in this example, only in a later section (Sect. 4.2) of the paper. The prior probability of failure was taken into account in a parametric study representing various damage severities according to Table 2.

The results of the parametric study for the fundamental case are shown in Table 3. For low criticality and minor damage levels 'No Assessment' is the preferred option, because the expected cost of improvement in safety confidence is too high compared to the consequences of 'Do nothing'. In practice, some non-invasive actions such as limiting vehicle weights or speeds for the bridge could be considered for this case. If the damage severity and/or criticality is increased, then an assessment method may be preferred. The exact method to be chosen will depend on its cost and sensitivity/specificity (i.e. sample likelihood). From Table 3 it can be seen that there is a region where 'Assessment 1' is the preferred option over 'Assessment 2'. If the damage severity and/or criticality is increased further, then 'Assessment 2' becomes the preferred option. Finally, when both damage severity and bridge criticality are high, the expected damage costs exceed repair costs, thus making any assessment unnecessary ('No Assessment'); in this case, repair becomes the preferred action.

\subsection{Successive assessment}

As it has been shown in the the previous subsection, obtaining SHI through condition assessments is a promising way to reduce long-term maintenance costs (as a result of decreased expected utilities) without the need for costly invasive actions such as repair. It thus seems logical that further improvements might be realized if the assessments are used successively, before the final decision about intervention is made. This approach is illustrated with a decision tree in Fig. 3. In considering this type of decision problem, it should be mentioned that the results obtained will, just as with the assessment methods themselves, provide optimal decisions in sequence. In other words, an analysis can evaluate whether no assessment should be made (i.e. repair or do nothing) or if the first, of possibly many, assessments should be made. Decisions concerning subsequent actions
Table 3 Optimal assessments for the fundamental case (with expected utilities)

\begin{tabular}{lllll}
\hline Bridge & Damage severity & & \\
\cline { 2 - 4 } criticality & Minor & Moderate & Significant & Severe \\
\hline Low & No Ass. (-0.1) & No Ass. (-1) & No Ass. (-10) & No Ass. (-100) \\
Mid-low & No Ass. (-1) & No Ass. (-10) & No Ass. (-100) & Ass. 1 (-218) \\
Mid-high & No Ass. $(-10)$ & No Ass. (-100) & Ass. 1 (-210.8) & Ass. 2 (-319.8) \\
High & No Ass. $(-100)$ & Ass. 1 (-210.08) & Ass. 2 (-310.98) & No Ass. (-1000) \\
\hline
\end{tabular}


Fig. 3 Decision tree with several assessment levels

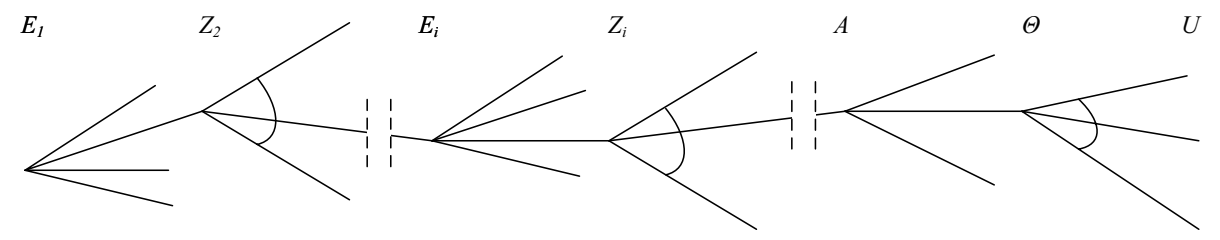

$E_{1}$

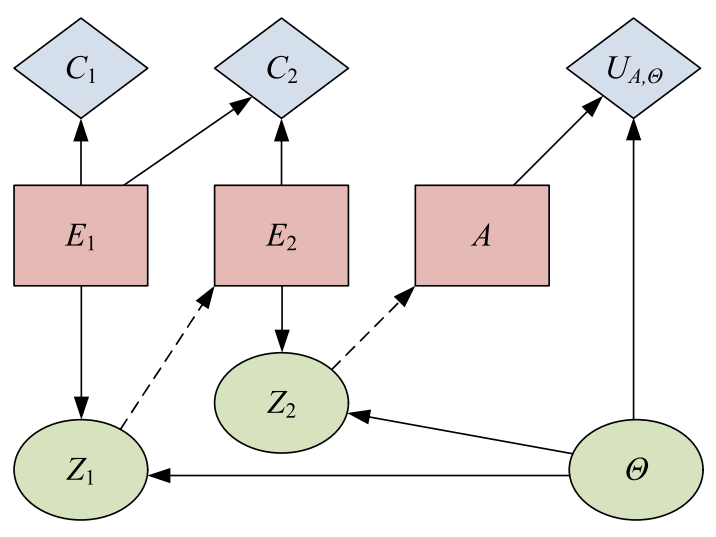

Fig. 4 Influence diagram for the successive case

requires results from the first assessment as input; i.e. if a negative result is given, then additional assessments (or other actions) may help while a positive result may yield that not further assessment is needed. This aspect is reflective of how such decisions would be made in practice and is thus to be expected.

For the first case, let us assume that two levels of assessments are applied, i.e. it is possible to opt for collecting SHI twice. The first decision is concerned with choosing between 'No Assessment' and 'Assessment 1' $\left(E_{1}=\{\right.$ No Assessment; Assessment 1$\}$ ), whereas the second decision (taken after the first) represents a choice between 'No Assessment' and 'Assessment 2' $\left(E_{2}=\{\right.$ No Assessment; Assessment 2$\left.\}\right)$. The related influence diagram is depicted in Fig. 4. It would be possible to make the decision problem more general, i.e. all options are available at $\left(E_{1}\right)$ and all or only the remaining options are available at $\left(E_{2}\right)$. However, the suggested strategy, i.e. considering the cheapest and less efficient method first, is a good representation of how decisions are made in current practice. Furthermore, limiting the number of options reduces computational complexity, which is also an important aspect regarding practical applicability. Nevertheless, it should be noted that higher gains in terms of expected utilities might be realized using a more general strategy.

The results of the analysis, which are shown in Table 4, indicated that there is an inherent value (in terms of increased expected benefit) with utilizing successive assessments. To start, the table shows the optimal first decisions for the same scenarios considered in the previous fundamental case (Table 3). In all cases it can be seen that either 'No Assessment' is made or 'Assessment 1' should be made first. Considering then the expected benefit evaluated for these decisions, and comparing them with the results from the fundamental case, an added value of successive assessments is determined (shown within parentheses in Table 4). For example, in the case of moderate damage severity and high bridge criticality, the optimal decision is to apply decision 'Assessment 1' first, and possibly 'Assessment 2' thereafter (depending on which results were obtained in the first assessment), and the overall expected benefit was 33\% higher than for the fundamental case (even though the optimal decision for the fundamental case was also to apply 'Assessment 1'). This indicates an inherent value of successive assessment which requires further study.

\section{Further examples}

\subsection{High repair costs}

As seen from the previous example, the optimal assessment strategy and the expected cost reduction through informed decision making depend on several factors. These include prior expectations about damage severity and bridge criticality (i.e. the relative costs of failure and repair) as well as the cost and the sample likelihoods (sensitivity/specificity of assessment options). Therefore, general consequences are difficult to draw based on the previous results. It is, however,
Table 4 Optimal first assessments if they are applied successively (and relative gain in expected utilities)

\begin{tabular}{|c|c|c|c|c|}
\hline \multirow{2}{*}{$\begin{array}{l}\text { Bridge } \\
\text { criticality }\end{array}$} & \multicolumn{4}{|l|}{ Damage severity } \\
\hline & Minor & Moderate & Significant & Severe \\
\hline Low & No Ass. (0\%) & No Ass. (0\%) & No Ass. (0\%) & Ass. 1 (47.60\%) \\
\hline Mid-low & No Ass. $(0 \%)$ & No Ass (0\%) & Ass. 1 (57.05\%) & Ass. 1 (30.96\%) \\
\hline Mid-high & No Ass. (0\%) & Ass. 1 (58.00\%) & Ass. 1 (33.09\%) & Ass. $1(1.09 \%)$ \\
\hline High & Ass. 1 (58.09\%) & Ass. $1(3.31 \%)$ & Ass. $1(0.40 \%)$ & Ass. 1 (59.37\%) \\
\hline
\end{tabular}


Table 5 Optimal non-successive assessments for increased repair costs (with expected utilities)

\begin{tabular}{lllll}
\hline $\begin{array}{l}\text { Bridge } \\
\text { criticality }\end{array}$ & Damage severity & & & \\
\cline { 2 - 5 } Minor & Moderate & Significant & Severe \\
\hline Low & No Ass. (-1) & No Ass. (-10) & No Ass. (-100) & Ass. 2 (-408) \\
Mid-low & No Ass. (-10) & No Ass. (-100) & Ass. 2 (-319.8) & Ass. 2 (-498) \\
Mid-high & No Ass. (-100) & Ass. 2 (-310.98) & Ass. 2 (-409.8) & Ass. 2 (-1398) \\
High & Ass. 2 $(-310.1)$ & Ass. 2 $(-400.98)$ & Ass. 2 (-1309.8) & No Ass. (-10000) \\
\hline
\end{tabular}

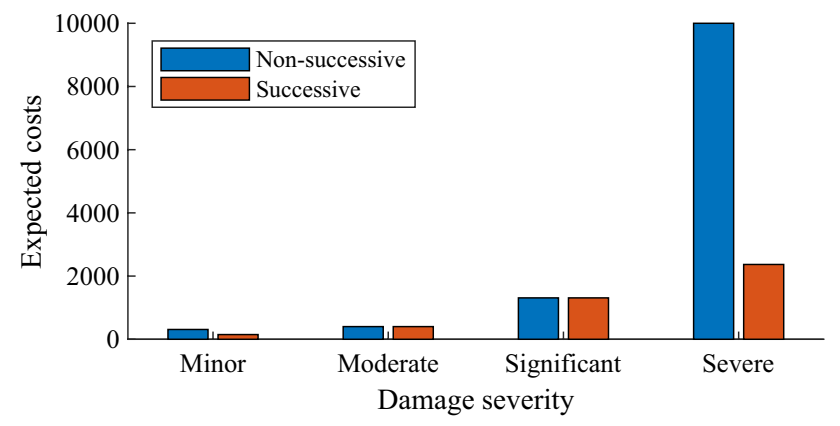

Fig. 5 Expected utilities (expressed as expected costs) for high bridge criticalty (and expensive repair), non-successive vs successive assessment (2 levels)

clear that the successive strategy is superior over the nonsuccessive one in terms of expected utility. This is because the successive case encompasses the possibility of the fundamental case and the value of SHI cannot be negative; an assumption which is typically true [16]. As a comparison, the next example shows how the additional VoI gained by successive assessment strategy changes if the repair costs are 10 times higher than in the previous section, i.e. $C_{r}=10,000$.

The results for the single choice decision (non-successive case) are given in Table 5 in terms of optimal assessment choices and expected utilities (in parentheses). To visualise the differences between the expected utilities using non-successive and successive case, bar charts are plotted in Fig. 5 for the case of high bridge criticality. In the figure, negative utilities are expressed as costs (positive values) for an easier interpretation, i.e. lower cost means higher utility. The difference between the bars can be interpreted as the net VoI according to Eq. 2. It is interesting to observe that in this case 'Assessment 1' is never preferred over 'Assessment 2' as it is seems worth paying for "better" SHI before any decision on costly intervention is to be made.

The overall results of the successive assessment are presented in Table 6, i.e. the first assessment choices and the relative expected utility gains (in parentheses). As soon as successive strategy is applied the significance of 'Assessment 1 ' becomes clear again. In several damage severitybridge criticality combinations it is useful to start the process with 'Assessment 1' and decide on the application of 'Assessment 2' based on its actual outcome. It should be mentioned there are cases where no assessment is required at the first level, but at the second level 'Assessment 2' is the preferred option (these are indicated with ${ }^{\mathrm{a}}$ in Table 6).

\subsection{Additional leve}

If successive strategies are indeed superior to non-successive ones, it follows that additional levels of assessment could lead to even better utilization of SHI and thus potentially further reduce maintenance costs. Consider the same example as before including the possibility of a third assessment method and a third level of assessment. Assume that the cost of 'Assessment 3' is 500 monetary unit and the sample likelihoods are according to the last columns of Table 1 .

First, the non-successive strategy is investigated to see if there are some damage severity-bridge criticality combinations for which 'Assessment 3' is a useful options. The results indicate that they could be beneficial if both damage severity and bridge criticality are high, as indicated in Table 7. Differences from the 2-level case results are marked by ${ }^{\mathrm{a}}$.

As a next step, we consider that the three assessment options are applied successively and determine the optimal
Table 6 Optimal first assessments with increased repair costs (and relative gain in expected utilities)

\begin{tabular}{|c|c|c|c|c|}
\hline \multirow{2}{*}{$\begin{array}{l}\text { Bridge } \\
\text { criticality }\end{array}$} & \multicolumn{4}{|l|}{ Damage severity } \\
\hline & Minor & Moderate & Significant & Severe \\
\hline Low & No Ass. $(0 \%)$ & No Ass. (0\%) & Ass. 1 (40.04\%) & Ass. $1(41.27 \%)$ \\
\hline Mid-low & No Ass. $(0 \%)$ & Ass $1(48.19 \%)$ & Ass. $1(50.58 \%)$ & No Ass. ${ }^{a}(0 \%)$ \\
\hline Mid-high & Ass. 1 (49.01\%) & Ass. 1 (51.80\%) & No Ass. ${ }^{\mathrm{a}}(0 \%)$ & No Ass. ${ }^{a}(0 \%)$ \\
\hline High & Ass. 1 (51.92\%) & No Ass. ${ }^{a}(0 \%)$ & No Ass. ${ }^{a}(0 \%)$ & Ass. 1 (76.3\%) \\
\hline
\end{tabular}

${ }^{\mathrm{a}}$ Then Assessment 2 
Table 7 Optimal nonsuccessive assessments for three assessment options and expensive repair (with expected utilities)

Table 8 Optimal first assessments for three levels (and relative gain in expected utility)

\begin{tabular}{|c|c|c|c|c|}
\hline \multirow{2}{*}{$\begin{array}{l}\text { Bridge } \\
\text { criticality }\end{array}$} & \multicolumn{4}{|l|}{ Damage severity } \\
\hline & Minor & Moderate & Significant & Severe \\
\hline Low & No Ass. $(-1)$ & No Ass. $(-10)$ & No Ass. $(-100)$ & Ass. 2 (-408) \\
\hline Mid-low & No Ass. $(-10)$ & No Ass. $(-100)$ & Ass $2(-319.8)$ & Ass. 2 (-498) \\
\hline Mid-high & No Ass. $(-100)$ & Ass. $2(-310.98)$ & Ass. $2(-409.8)$ & Ass. $3^{\mathrm{a}}(-709.8)$ \\
\hline High & Ass. $2(-310.1)$ & Ass. 2 (-400.98) & Ass. $3^{\mathrm{a}}(619.98)$ & Ass. $3^{\mathrm{a}}(-1609.8)$ \\
\hline
\end{tabular}

${ }^{\text {a}}$ Differs from the 2-option case

\begin{tabular}{|c|c|c|c|c|}
\hline \multirow{2}{*}{$\begin{array}{l}\text { Bridge } \\
\text { criticality }\end{array}$} & \multicolumn{4}{|l|}{ Damage severity } \\
\hline & Minor & Moderate & Significant & Severe \\
\hline Low & No Ass. (0) & No Ass. (0) & Ass. 1 (48.99\%) & Ass. 1 (42.29\%) \\
\hline Mid-low & No Ass. (0) & Ass $1(57.55 \%)$ & Ass. $1(53.12 \%)$ & Ass. $1^{\mathrm{a}}(26.06 \%)$ \\
\hline Mid-high & Ass. 1 (58.41\%) & Ass. 1 (54.55\%) & Ass. $1^{\text {a }}(32.69 \%)$ & Ass. $1^{\mathrm{a}}(31.58 \%)$ \\
\hline High & Ass. 1 (54.69\%) & Ass. $1^{\mathrm{a}}(33.51 \%)$ & Ass. $1^{\mathrm{a}}(36.15 \%)$ & Ass. $1(53.07 \%)$ \\
\hline
\end{tabular}

${ }^{\text {a }}$ Differs from the 2-level case

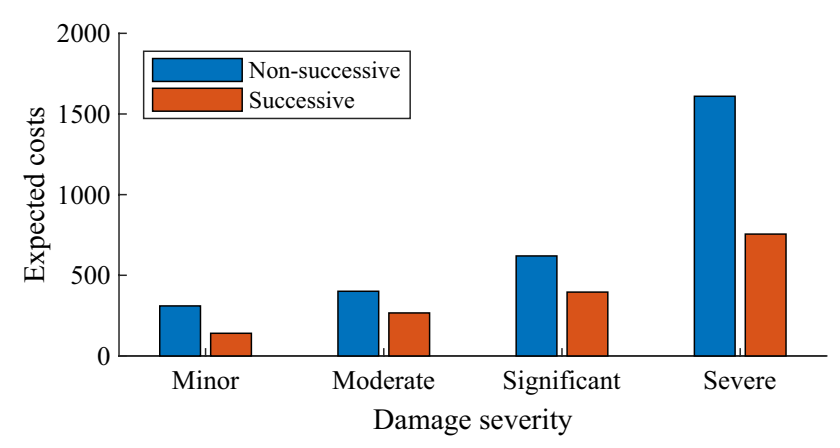

Fig. 6 Expected utilities (expressed as expected costs) for high bridge criticalty (and expensive repair), non-successive vs successive assessment (3 levels)

first decision together with the additional gain in expected utility (compared to non-successive assessments) as in the previous example. The results are given in Table 8, indicating (by ${ }^{a}$ ) where the first option differs from the two-level case. For a visualisation, a comparison of the expected utilities (shown as costs) for the high bridge criticality case is plotted in Fig. 6. Compared with Fig. 5, it can be seen that the relative reduction of expected costs is higher in the case of three successive assessment than for just two. This reiterates a previous point concerning the added value of information from additional assessments.

In the current example, it is also interesting to calculate the expected utility gain between the successive strategy with 3 vs 2 levels of assessment:
$\operatorname{VoI}($ additional assessment $)=u^{*}(3$ levels $)-u^{*}(2$ levels $)$

This extra VoI obtained by the additional assessment level is shown in Fig. 7 (left). To gain better insights on the relative utility gain expected by the additional assessment level, a normalized value of the extra $\mathrm{VoI}$ is calculated according to:

$\overline{\mathrm{VoI}}($ additional assessment $)=\frac{u^{*}(3 \text { levels })-u^{*}(2 \text { levels })}{u^{*}(2 \text { levels })}$

These normalized values, $\overline{\mathrm{VoI}}$, are given in Fig. 7 (right). In both Table 8 and Fig. 7 it can be seen that there is sometimes an inherent value with applying successive assessments and that this value increases with the number of assessments considered. The actual increase is a function of the damage severity and bridge criticality, where a higher value of both yields a higher value of information. Another result from all of the previous examples is that it is always optimal to choose the cheapest assessment method first (if a method is preferable over the other actions).

\section{Discussion and conclusions}

The major conclusion of the presented study is that successive assessment strategies have an inherent value over non-successive assessments due to the additional possibility of updating knowledge about the structural condition. This knowledge then provides better foundation for maintenance decisions. The results in this paper indicate that successive assessment is always a better option. Furthermore, additional assessment levels can provide more value of information 
Fig. 7 Expected utility gain by the additional assessment level: absolute values, $\mathrm{VoI}$ (left) and relative values, $\overline{V o I}$ (right)

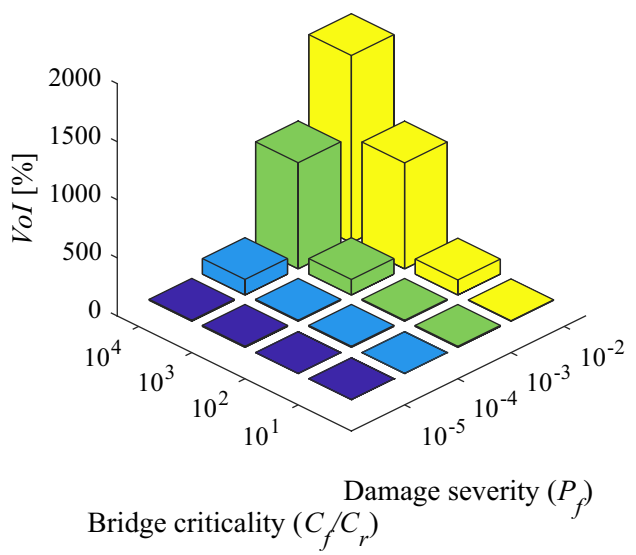

while the amount of value added depends on damage severity and bridge criticality; if both are high, the additional VoI also increases. In other words, important bridges in poor condition are worth investigating in several successive steps.

In the current paper, there are several assumptions and their impacts shall be discussed. First, it is assumed that the assessments carried out for a certain bridge are independent from each other. It is unclear if such an assumption can be perfectly true for any bridge although higher degrees of independence are possible if the condition assessments are carried out in the proper way. Even if the option of using the same method multiple times was possible, in practice it should be done in a way that allows for the best use of information to be obtained. For example, if the decision maker would use the same non-destructive measurement twice, she should not use the exact same instrument; if the assessment to be used multiple times refers to a complete structural analysis, it is better to be carried out by different persons, etc. Redoing the same tests would, however be appropriate if one suspects that the results are corrupt. Otherwise, it would be more appropriate to collect information about something else, or perhaps collect more samples to decrease the epistemic uncertainty. There are typically several uncertain parameters, thus measurements of other quantities would often be more adequate than applying an additional assessment level.

Second, it is assumed that each additional assessment level refers to a more sensitive and more specific method. Thus, if one could, theoretically, include infinite number of levels, the information to be obtained would converge to perfect information thus the state of nature would (almost) be known by certainty. Based on (nearly) prefect knowledge, actually the best intervention could be selected.

Third, it is assumed that the value of information is nonnegative. There are some special cases where it is not necessarily true [16]. In practice, this could be the case, for example, if the decision maker expects that the assessment would uncover some unknown issues that they would rather like to hide. In that case, however, the decision maker is probably not fully responsible for all the risks involved, e.g. due to mitigation through insurance.

It should be highlighted that when referring to possible reduction of maintenance costs, this is meant in an expected sense. That is, if one systematically and consistently applies successive assessments for a large number of bridges (or for a certain bridge for a sufficiently long period of time) the overall maintenance costs will be reduced in the long run compared to single choice decisions. It does not mean, however, that some decisions will not be sub-optimal and that the expected utility gains will always be realized.

In practice the presented framework should be applied for a case by case basis. The added value of information for successive assessment is dependent on the priors and sample likelihoods as well as various costs. In practical cases, if assessments are deemed useful, typically a relatively cheap assessment method should be applied first. What is done after that will then depend on the results of the first assessment to be used for calculating the posterior failure probability.

Acknowledgements Open access funding provided by RISE Research Institutes of Sweden. Swedish Transport Administration (Trafikverket) (Grant no.TRV 2019/46154) and the strategic innovation programme InfraSweden2030 (Grant no. 2019-01162), a joint effort of Sweden's Innovation Agency (Vinnova), the Swedish Research Council (Formas) and the Swedish Energy Agency (Energimyndigheten).

Open Access This article is licensed under a Creative Commons Attribution 4.0 International License, which permits use, sharing, adaptation, distribution and reproduction in any medium or format, as long as you give appropriate credit to the original author(s) and the source, provide a link to the Creative Commons licence, and indicate if changes were made. The images or other third party material in this article are included in the article's Creative Commons licence, unless indicated otherwise in a credit line to the material. If material is not included in the article's Creative Commons licence and your intended use is not permitted by statutory regulation or exceeds the permitted use, you will 
need to obtain permission directly from the copyright holder. To view a copy of this licence, visit http://creativecommons.org/licenses/by/4.0/.

\section{References}

1. Benjamin J, Cornell C (2014) Probability, statistics, and decision for civil engineers. Dover books on engineering. Dover Publications, New York

2. Björnsson I, Larsson Ivanov O, Leander J, Honfi D (2019) Decision support framework for bridge condition assessments. Struct Saf 81:101874

3. Woodward RJ, Cullington DW, Daly AF, Vassie PR, Haardt P, Kashner R et al (2001) Bridge management in Europe (BRIME) deliverable D14. Final Report PL97-2220. TRL Ltd, Wokingham

4. Calvi G, Moratti M, O'Reilly G, Scattarreggia N, Monteiro R, Malomo D, Calvi P, Pinho R (2019) Once upon a time in Italy: the tale of the Morandi Bridge. Struct Eng Int 29(2):198-217

5. Gkoumas K, Marques dos Santos F, Van Balen M, Tsakalidis A, Ortega Horteland A, Grosso M etal (2019) Research and innovation in bridge maintenance, inspection and monitoring-a European perspective based on the transport research and innovation monitoring and information system (TRIMIS). Publications Office of the European Union, Luxembourg

6. Goulet JA, Kiureghian AD, Li B (2015) Pre-posterior optimization of sequence of measurement and intervention actions under structural reliability constraint. Struct Saf 52:1-9

7. Honfi D, Leander J, Björnsson I (2017) Decision support for bridge condition assessment. In: In: 4th international conference on smart monitoring, assessment and rehabilitation of civil structures (SMAR 2017), Zürich, Switzerland, 13-15, September 2017, paper 323

8. Howard RA (1966) Information value theory. IEEE Trans Syst Sci Cybern 2(1):22-26

9. Howard RA (1967) Value of information lotteries. IEEE Trans Syst Sci Cybern 3(1):54-60

10. ISO (2010) ISO 13822:2010, Bases for design of structuresassessment of existing structures. International Organization for Standardization, Geneva

11. Khamis HJ (1987) A statistics refresher: tests of hypothesis and diagnostic test evaluation. J Diagn Med Sonogr 3(3):123-129

12. Kühn B, Lukić M, Nussbaumer A, Günther HP, Helmerich R, Herion $\mathrm{S}$ et al (2008) Assessment of existing steel structures: recommendations for estimation of remaining fatigue life. In: Prepared under the JRC-ECCS cooperation agreement for the evolution of Eurocode 3 (programme of CEN/TC 250). Office for Official Publications of the European Communities, Luxembourg
13. Leander J, Honfi D, Ivanov OL, Björnsson I (2018) A decision support framework for fatigue assessment of steel bridges. Eng Fail Anal 91:306-314

14. Ponssard JP (1976) On the concept of the value of information in competitive situations. Manag Sci 22(7):739-747

15. Pozzi M, Der Kiureghian A (2011) Assessing the value of information for long-term structural health monitoring. In: Kundu T (ed) Health monitoring of structural and biological systems 2011, vol 7984. SPIE, pp 812-825

16. Pozzi M, Malings C, Minca AC (2017) Negative value of information in systems' maintenance. In: Bucher C, Ellingwood BR, Frangopol DM (eds) Proceedings of 12th international conference on structural safety and reliability (ICOSSAR), 2017, pp 3339-3346

17. Raiffa H, Schlaifer R (2000) Applied statistical decision theory. Wiley Classics Library. Wiley, New York

18. SAMCO: Final report, f08a guideline for the assessment of existing structures (2006)

19. Shu J, Honfi D, Plos M, Zandi K, Magnusson J (2019) Assessment of a cantilever bridge deck slab using multi-level assessment strategy and decision support framework. Eng Struct 200:109666

20. Sousa H, Santos LO, Chryssanthopoulos M (2019) Quantifying monitoring requirements for predicting creep deformations through bayesian updating methods. Struct Saf 76:40-50

21. Straub D (2014) Value of information analysis with structural reliability methods. Struct Saf 49:75-85 Special Issue In Honor of Professor Wilson H. Tang

22. Sustainable Bridges (2007) Guideline for load and resistance assessment of existing European railway bridges. Sustainable Bridges - Assessment for future traffic demands and longer lives. http://www.sustainablebridges.net

23. Thöns S (2018) On the value of monitoring information for the structural integrity and risk management. Comput Aided Civ Infrastruct Eng 33(1):79-94

24. Trafikverket: Batman rapport—broars ålderfördelning. (2019). https://webapp.trafikverket.se/VNapps/vv/webhybris/30.Fakta /32.03 Broars åldersfördelning.vns) Accessed 12 Dec 2019

25. U.S. Department of Transportation, Federal Highway Administration: 2015 Status of the Nation's Highways, bridges and transit: conditions and performance (2015)

26. Wilson ECF (2015) A practical guide to value of information analysis. PharmacoEconomics 33(2):105-121

Publisher's Note Springer Nature remains neutral with regard to jurisdictional claims in published maps and institutional affiliations. 